\title{
Engineering Chiral Structures Through Strain Release: Electron Tomography Study of Twisted Nanowires
}

\author{
Xiaohui Song ${ }^{1}$, Alex Bruefach ${ }^{2}$, Philipp M. Pelz ${ }^{2}$, Hannah Devyldere ${ }^{3}$, Mary Scott ${ }^{1,2}$ \\ 1. The Molecular Foundry, Lawrence Berkeley National Lab, Berkeley, CA, USA. \\ 2. Department of Materials Science and Engineering, University of California, Berkeley, Berkeley, CA, \\ USA. \\ 3. Current affiliation: Thermo Fischer Scientific, Guilford, Connecticut, USA.
}

Defects and strain play a strong role in material functionality on the nanoscale, but they are also important in directing the growth of many nanomaterials. For example, an axial screw dislocation is behind the asymmetric growth that creates many types of nanowires $[1,2]$. In general, asymmetric nanomaterials are of technological interest due to their novel optoelectronic properties. Beyond individual properties, chiral and helical structures allow one to tune parameters such as diameter, helix pitch and spacing between individual helices. These hierarchical length scales create the capability to encode complementary properties in a single nanomaterial, such as tuning catalytic and optical properties for idealized photocatalysis. Coupled optical and plasmonic properties depend on material spacing, so the ability to create interlocking and tunable morphologies of helical structures holds great promise. However, before these materials can be widely implemented in technological applications, a better understanding of the factors that govern their synthetic routes must be developed.

Boerdijk-Coxeter-Bernal (BCB) helical nanowires are polycrystalline nanowire structures. The BoerdijkCoxeter helix [3], Fig. 1, is formed from tetrahedra with shared faces, with vertices following a line (Fig. 1d). It has been previously demonstrated that nanostructures with the BCB structure can be obtained by synthesizing a bimetallic AuAg nanowires from silver and gold salts [3, 4]. Although the microstructure of these nanowires are complex and polycrystalline, the overall morphology is essentially straight. However, when a shell of single atomic species is subsequently grown on the straight nanowire core, twisting and helical structures can be obtained [4, 5]. Figure 3 illustrates this process for a Pd shell grown on the AuAg NW seed. While there are many methods to synthesize twisted and helical structures, BCBbased wires offer the advantage in flexibility in choice of the atomic species of the shell, with a variety of FCC metal shells being demonstrated. The relationship to other nanoscale structures, as well as the origin of chirality in these structures, is also of great interest.

Transmission electron microscopy (TEM) and scanning transmission electron microscopy (STEM) have been widely applied in nanoscale science, helping to answer many key questions of morphology and lattice information at the nanoscale. Of key interest is the structural evolution of nanomaterials during synthesis, and how to direct growth for desired features. In particular, the interior grain structure, core-shell lattice mismatch, presence of defects, and any alloying between core and shell in the BCB-based nanowires could have a strong effect on the final structure. Elucidating these relationships would enable tunable design of these materials. Because these nanowires have complex polycrystalline core-shell structures, twodimensional projection images are not enough.

Here, we present a detailed three-dimensional study of BCB-based twisted and helical nanowires. The proposed mechanism for the massive morphological transition that BCB nanowires undergo is a strain release mechanism. We find that upon varying the lattice mismatch between the core alloy and the shell 
material, we obtain different twisting behavior in the nanowires. Changing the relative concentration of straight nanowires and shell precursor material during shell deposition results in various morphologies, including multiple-strand helices and straight nanowires with complex polycrystalline shells. In the case of the straight nanowires with polycrystalline cores, high resolution tomography reveals the interior grain structure maintains a polycrystalline structure. Ultimately, this information can be used to better control the synthesis of these materials for tunable helical nanostructures with desired optoelectronic properties $[6]$.

\section{References:}

[1] Bierman, Matthew J. et al., Science 320.5879 (2008), p. 1060.

[2] Zhu, Jia, et al., Nature nanotechnology 3.8 (2008), p. 477

[3] J. J. Velázquez-Salazar et al., ACS nano 5(8) (2011), p. 6272.

[4] Y. Wang et al., Journal of the American Chemical Society 133(50) (2011), p. 20060.

[5] Y. Wang et al., Chemical Society Reviews 42(7) (2013), p. 2930.

[6] Work at the Molecular Foundry was supported by the Office of Science, Office of Basic Energy Sciences, of the U.S. Department of Energy under Contract No. DE-AC02-05CH11231.

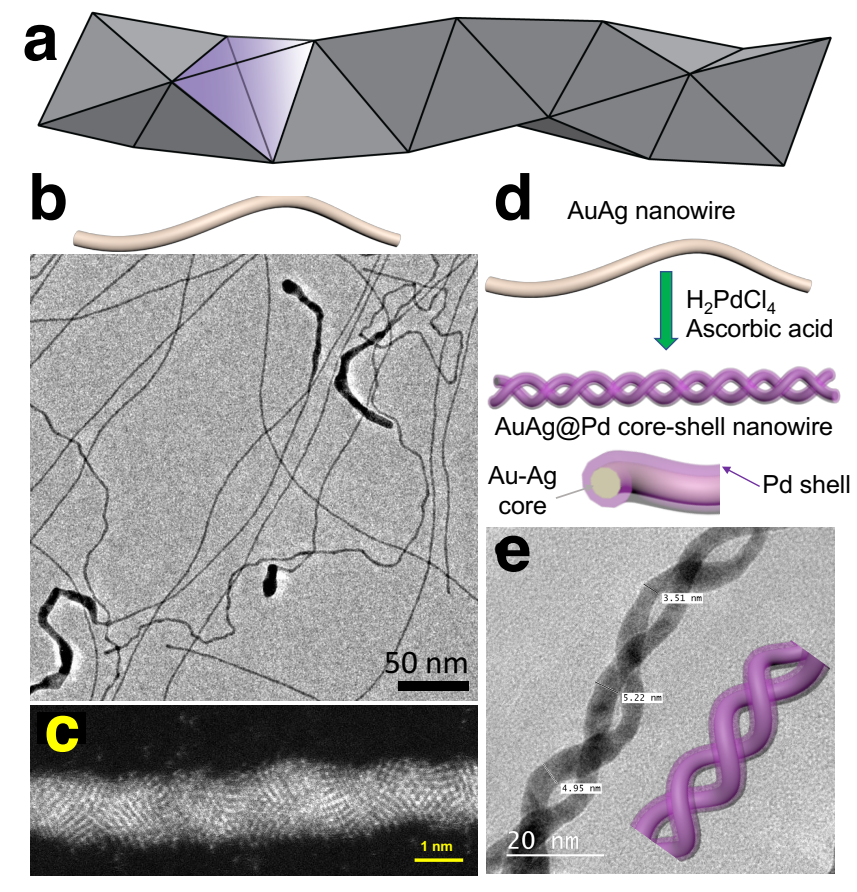

Figure 1. BCB nanowire twisting. (a) The ideal BCB helix structure (b) Straight, AuAg alloy BCB nanowires. (c) The atomic resolution structure of the wires in (b) demonstrate decahedral packing consistent with the BCB structure. (d) Upon coating with Pd, the wires twist into a double helix structure. (e) TEM image of the coated wires after twisting. 